\title{
Long-Term Effects of Dexmedetomidine versus Propofol During the Implantation of a Neurostimulator-A Post-Trial Follow-Up Analysis
}

\author{
Feline FJA ter Bruggen (D' \\ W Ken Redekop (iD ${ }^{2}$ \\ Dirk L Stronks' \\ Frank JPM Huygen' \\ 'Department of Anesthesiology, Center \\ for Pain Medicine, Erasmus MC, \\ Rotterdam, the Netherlands; \\ ${ }^{2}$ Department of Health Technology \\ Assessment, Erasmus School of Health \\ Policy \& Management, Erasmus University \\ Rotterdam, Rotterdam, the Netherlands
}

\begin{abstract}
Objective: The success of neurostimulation depends partly on the amount of coverage of the neurostimulation-induced paresthesia of the painful area. This is often achieved by asking feedback from patients intraoperatively. If sedation analgesia is used, it is important that the patient is comfortable during sedation and easily arousable. If the patient is not well sedated or experiences residual effects of the sedation during testing, this can directly influence the ideal placement of the leads and indirectly the long-term effect of the treatment. It is our hypothesis that the quality of the sedation is directly coupled to the adequacy of lead placement and in this way in the result of the treatment. Dexmedetomidine is known for its easy production of arousable sedation. The aim of the present study was to compare the long-term effect of using dexmedetomidine versus propofol during the implantation of a neurostimulator.
\end{abstract}

Materials and Methods: This is a post-trial follow-up analysis of the DexMedPro cohort. The primary outcome was global perceived effect (GPE). The secondary outcomes were the course of pain intensity, the emotional and physical functioning at the time of follow-up, and the course of neurostimulation treatment. In this study, we used the patient satisfaction with sedation as a measure for sedation quality.

Results: Regarding the GPE, no statistically significant differences were found between the experimental groups in either subscale (ie, recovery $(\mathrm{p}=0.82)$ and satisfaction with the neurostimulation treatment at follow-up $(\mathrm{p}=0.06))$. The same was found regarding the secondary parameters. A correlation was found between patient satisfaction with sedation during the lead implantation (side effects and procedural recall) and satisfaction at follow-up. Conclusion: Regarding the long-term efficacy of neurostimulation treatment, no statistically significant differences were found between the dexmedetomidine and the propofol group. We observed a trend towards greater satisfaction with the neurostimulation treatment at followup in the dexmedetomidine group, compared to the propofol group.

Keywords: dexmedetomidine, propofol, chronic pain, neurostimulation, follow-up, retrospective studies

\section{Introduction}

Spinal cord neurostimulation is a proven cost-effective treatment of chronic pain. ${ }^{1-3}$ The success of this intervention partially relies on stimulation-induced paresthesia and the extent of its coverage of the painful area(s). ${ }^{4}$ Although, more recently, anatomical lead placement, which does not rely on paresthesia, has received
Correspondence: Feline FJA ter Bruggen Tel +3I 640962334

Email ftbruggen@gmail.com;

f.terbruggen@erasmusmc.nl 
increasing attention with comparable outcomes, optimal paresthesia coverage is still often achieved by seeking feedback from patients intraoperatively. ${ }^{5}$ In our center, and elsewhere across Europe, the most common method of implantation is to apply sedation analgesia and wake up the patient during a test stimulation.

Optimal paresthesia coverage depends on the combination of stimulation parameters and the location of the lead(s) and it is achieved by using patients' feedback. Placement of the leads is easier with an adequate sedation. However, reliable feedback can be obtained if the patient is arousable - preferably easily. Different sedation analgesia strategies can facilitate this in varying degrees.

The most commonly used sedation-analgesia regimen during a neurostimulation procedure in the Netherlands is the combination of propofol-remifentanil. ${ }^{6}$ Propofol is frequently used partly because anesthesiologists have much experience with it. It provides a smooth and rapid induction and easily controllable and stable continuation of sedation, and its cost is relatively low. ${ }^{7}$ However, because propofol acts on the GABA receptors, difficulties may arise with regard to obtaining reliable feedback immediately after waking, due to the drowsiness of the patient.

Dexmedetomidine is an alpha-2 agonist with sedative, analgesic, and anxiolytic properties, known for its ability to produce arousable sedation in a moderately sedated patient, with spontaneous ventilation. ${ }^{8}$ Previous studies have reported promising results regarding the use of dexmedetomidine in awake procedures, such as during an awake craniotomy and during the surgical implantation of paddle lead electrodes for neurostimulation through a laminectomy. ${ }^{9,10}$ In the DexMedPro trial, we compared dexmedetomidine with propofol during the implantation of a neurostimulator - as a sedative agent - in terms of patient satisfaction and safety. The results indicate higher satisfaction with sedation and an easier production of arousable sedation of patients who received dexmedetomidine, compared to the propofol group. The results indicated a safe and stable situation for both patient groups during the procedure. ${ }^{11}$

A sedation that is too shallow can cause discomfort for the patient and the implantation team, which is to the detriment of the placement technique of the leads. A poorly awake patient during testing can lead to an inadequate assessment of the position of the leads. Both can have a negative effect on the results in the long term. In other words, it is presumed that a more controllable agent, such as dexmedetomidine leads to better sedation and better arousability and indirect to better lead placement with a better long-term effect. Therefore, our hypothesis was that a high-quality sedation regimen (dexmedetomidine or propofol sedation), indicated by a subjective outcome measurement (ie, patient satisfaction with sedation) and an objective measurement (easy arousability during the intervention) could have a long-term impact on pain intensity and the emotional and physical functioning of the patients. Hence, the aim of this retrospective study was to examine the extent to which longterm efficacy is associated with the sedation regimen (dexmedetomidine versus propofol) during the implantation of a neurostimulator. The outcome parameters were global perceived effect, pain course, physical and emotional functioning, and course of neurostimulation treatment in terms of complications.

\section{Materials and Methods}

This is a post-trial follow-up (PTFU) study of the DexMedPro trial (October 2015-April 2018; NL52755.078.15). This study was approved by the medical ethical committee of the Erasmus Medical Center in Rotterdam in March 2018.

\section{Study Design}

A retrospective cohort study.

\section{Subjects and Treatment}

Sixty-nine patients with an indication of a spinal cord neurostimulator were included in our previous DexMedPro trial. The aim of that trial was to examine whether the use of propofol versus dexmedetomidine as a sedative, leads to differences regarding patient satisfaction, ease of arousable sedation, and hemodynamic variables, during the percutaneous lead implantation of a neurostimulator. Subjects underwent an SCS trial (1-2 weeks) or an all in one procedure. Trial success was defined by the implanter and defined as $50 \%$ or more pain relief. Spinal cord stimulation (Medtronic, Minneapolis, USA; ABBOTT, Plano TX, USA; Nevro, Redwood city, USA) with 1-2 octrodes, conventional $40-80 \mathrm{~Hz}$ stimulation, Burst $5 \mathrm{x} 500 \mathrm{~Hz} / 40 \mathrm{~Hz}$ or $10 \mathrm{KHz}$ respectively or DRG stimulation (ABBOTT, Plano TX, USA) 1-4 DRG quads, conventional 10-20 Hz, rechargeable or recharge free all to the implanters clinical decision. Details regarding intra operative and postoperative data, dose of medication, indication for procedure, duration of procedure, level of sedation of the drugs are provided in the article about the DexMedPro trial. ${ }^{10}$ 
The participants in this current PTFU study were those included in the DexMedPro trial and provided a written informed consent. Patients were excluded from current analysis if they had received an extra lead to the existing neurostimulation system and/or if they had had a negative trial period. Furthermore, patients who had undergone an explant were excluded as well.

\section{Outcome Parameters}

All patients were evaluated by a review of the routine clinical records from the first intake at the department of Pain Medicine of the Erasmus Medical Center. Patient records, patient demographics, pain diagnosis, and device specifics are to be found in the article about the DexMedPro trial.

The outcome parameters were measured a questionnaire administered by telephone in the period from January to February 2020. The primary outcome parameter was global perceived effect, measured by the global perceived effect questionnaire (GPE), see Table $1,{ }^{12}$ at the time of the telephone interview, regardless of the duration of therapy to date. The secondary outcome parameters were pain course (measured by the NRS pain score), emotional functioning (measured by the Hospital Anxiety Depression Scale [HADS]), physical functioning (measured by the Short Form Health Survey [RAND-36]),

Table I Seven-Point Scale of the Global Perceived Effect (GPE) Questionnaire

\begin{tabular}{|c|c|}
\hline Global Perceived Effect & \\
\hline $\begin{array}{l}\text { I. How would you rate the course of your } \\
\text { recovery since the start of this study? }\end{array}$ & $\begin{array}{l}\text { I. Completely } \\
\text { recovered } \\
\text { 2. Much improved } \\
\text { 3. Slightly improved } \\
\text { 4. Not changed } \\
\text { 5. Slightly worse } \\
\text { 6. Much worse } \\
\text { 7. Worse than ever }\end{array}$ \\
\hline $\begin{array}{l}\text { 2. How satisfied are you with the } \\
\text { neurostimulation treatment? }\end{array}$ & $\begin{array}{l}\text { I. Absolutely } \\
\text { satisfied } \\
\text { 2. Very satisfied } \\
\text { 3. Slightly satisfied } \\
\text { 4. Not satisfied nor } \\
\text { dissatisfied } \\
\text { 5. Somewhat } \\
\text { dissatisfied } \\
\text { 6. Very dissatisfied } \\
\text { 7. Absolutely } \\
\text { dissatisfied }\end{array}$ \\
\hline
\end{tabular}

Table 2 Long-Term Complications

\begin{tabular}{|l|}
\hline Complications \\
\hline Battery replacement \\
Complications requiring surgical intervention \\
Lead migration \\
Surgical site pain \\
Battery site pain \\
Fractured lead(s) \\
Lead revision \\
Infection \\
Seroma \\
Erosion \\
Epidural hematoma
\end{tabular}

and treatment course (measured by the occurrence of complications, see Table 2, based on the categorization used by Pope et al). ${ }^{13}$

\section{Statistical Analysis}

Descriptive statistics were used to determine the frequencies of the demographic variables and the outcome parameters and to describe measures of central tendency and variability, depending on the shape of their distribution.

Using the Shapiro Wilk test all parameters appeared to be skewly distributed. Therefore, the measure for the central tendency is presented as the median and the measure for the dispersion as the midspread, ie the InterQuartile Range (IQR: Q1 -Q3). To be able to analyse a difference over time in pain level between the experimental groups, the difference between the preoperative pain and the pain at the time of the interview was calculated. Differences between the experimental groups in the continuous parameters were analysed using the Independent-Samples Mann-Whitney U-Test. Spearman's rank correlation coefficient was used to test the degree of association between continuous variables. The difference in the proportion of the complications that did occur in the experimental groups was tested using the Fisher's exact test (twosided). The difference in the length of time between the implantation until the completion of the questionnaire may have had an effect on the level of outcome parameters. Therefore, we performed our analyses by dividing each experimental group into two groups using the median of the aforementioned length of time as the grouping criterion. Differences in the groups thus created were tested using the Independent-Samples Kruskal-Wallis Test. For all statistics, $\alpha$ was set at the traditional level of 0.05 level. Analyses were performed using IBM SPSS Statistics 22. 


\section{Results}

Following approval, 69 patients were included in the DexMedPro trial. If patients had had a negative trial period $(n=7)$, received an extra lead $(n=2)$, or died $(n=2)$, they were excluded from current analysis. Nine patients underwent an explant of the neurostimulation system. We have no data for five patients because they could not be reached by telephone ( 3 patients from the dexmedetomidine group and 2 patients from the propofol group). The remaining 44 patients were analysed.

The median number of days from implantation until completion of the questionnaire was 883 [IQR 706-1214] in the dexmedetomidine group and 837.5 [IQR 756.5$1104.5]$ in the propofol group $(p=0.73)$.

Regarding the subscale recovery of the GPE scale, the scores of the dexmedetomidine group (median 2.0 [IQR 2.0-3.0]) did not differ from those of the propofol group (median 2.0 [IQR 2.0-3.0]) $(\mathrm{p}=0.82)$. A trend was found in the subscale satisfaction in favor of the dexmedetomidine group (dexmedetomidine group median 2.0 [IQR 1.0-3.0] vs propofol group median 2.0 [IQR 2.0-3.0] $(\mathrm{p}=0.06)$ ).

We found a statistically significant negative correlation between the subscale satisfaction with neurostimulation treatment of the GPE and the subscale side effects of patient satisfaction with sedation index (PSSI) during the neurostimulation intervention administered at the end of the procedure and measured during the previous DexMedPro trial $(\mathrm{r}=-0.32 ; \mathrm{p}=0.035)$. This indicates that greater patient satisfaction in terms of side effects is associated with greater satisfaction with the effect of neurostimulation treatment reported at follow-up. Although not statistically significant, there was also a correlation identified between the subscale procedural recall of the PSSI and both subscales of the GPE (ie, satisfaction ( $\mathrm{r}=$ $-0.27 ; \mathrm{p}=0.08)$ and recovery $(\mathrm{r}=-0.30 ; \mathrm{p}=0.05))$. The data suggest that greater patient satisfaction with sedation (procedural recall) is associated with greater satisfaction reported at follow-up with the effect of the treatment.

No correlation was found between the number of titration adjustments and the reported overall effect of the procedure in terms of satisfaction and recovery (GPE).

The preoperative pain score was compared with the pain score at follow-up. The median pain difference in the dexmedetomidine group was 1.7 [IQR 0.9-3.9] versus 1.5 [IQR 0.75-3.4] in the propofol group. No significant difference was found between the experimental groups in the difference between the level of preoperative pain and that at the time of the follow up $(\mathrm{p}=0.45)$.

Regarding the depression subscale of the HADS questionnaire, the median score was 6.0 [IQR 3.0-10.0] in the dexmedetomidine group versus 4.0 [IQR 1.5-7.0] in the propofol group. This indicates a trend towards more depression complaints in the dexmedetomidine group $(\mathrm{p}=0.07)$.

The median score for the anxiety subscale was 4.0 [IQR 1.0-7.0] in the dexmedetomidine group versus 5.0 [IQR 2.5-7.0] in the propofol group, implying no statistically significant difference between the median scores between both groups $(p=0.95)$.

The same applied to the total median score on the HADS: 9.0 [IQR 5.0-15.0] in the dexmedetomidine group versus 8.0 [IQR 5.0-13.5]) in the propofol group $(\mathrm{p}=0.30)$.

Furthermore, no significant differences in the RAND36 domains were found between the experimental groups, see Table 3 .

The occurrence of complications was analysed from the day of implantation to the time at which the questionnaire was obtained or the moment of explant (see Table 4). There were no complications registered regarding infection, seroma, erosion, or epidural hematoma.

Table 3 Median and IQR (QI - Q3) for the Subscales of the RAND 36 by Experimental Group. Scale Scores Were Calculated Using the SF-36 Scoring System

\begin{tabular}{|l|c|c|c|}
\hline & Dexmedetomidine Group (n=23) & Propofol Group (n=21) & P \\
\hline Physical functioning & $50(20-60)$ & $35(22.5-60)$ & 0.84 \\
Social functioning & $50(25-62.5)$ & $50(37.5-68.75)$ & 0.64 \\
Role limitations - physical & $0(0-25)$ & $0(0-25)$ & 0.78 \\
Role limitations - emotional & $100(33.3-100)$ & $100(66.7-100)$ & 0.91 \\
Mental health & $80(64-88)$ & $80(66-84)$ & 0.47 \\
Vitality & $45(25-55)$ & $40(32.5-55)$ & 0.97 \\
Pain & $44.9(22.4-55.1)$ & $44.9(22.4-47.0)$ & 0.96 \\
General health & $45(25-65)$ & $40(30-60)$ & 0.48 \\
Change in health & $50(50-75)$ & $50(37.5-75)$ & 0.87 \\
\hline
\end{tabular}


Table 4 Complications That Occurred by Group, Multiple Complications Were Possible per Patient

\begin{tabular}{|l|c|c|}
\hline & Dexmedetomidine Group (n=23) & Propofol Group (n=2 I) \\
\hline Patients with complications & $17(74 \%)$ & $11(52 \%)$ \\
Battery replacement & $2(9 \%)$ & $2(10 \%)$ \\
Complications requiring surgical intervention & $3(13 \%)$ & $3(14 \%)$ \\
Migration & $4(17 \%)$ & $2(10 \%)$ \\
Surgical site pain & $5(22 \%)$ & $0(0 \%)$ \\
Battery site pain & $12(52 \%)$ & $10(48 \%)$ \\
Lead revision & $4(17)$ & $1(5 \%)$ \\
\hline
\end{tabular}

Median number of complications per patient in the dexmedetomidine group is 1.0 (IQR 0-2.0) and in the propofol group $1.0(\mathrm{IQR} 0-1.0)(\mathrm{p}=0.11)$. The proportion of patients with one or more complications did not differ significantly between the dexmedetomidine group and the propofol group $(\mathrm{p}=0.12)$.

The difference in the length of time between the implantation until the completion of the questionnaire may have had an effect on the level of outcome parameters. The results of these analyses did not differ from the previously performed.

\section{Discussion}

In the DexMedPro trial, the satisfaction of the patients who received dexmedetomidine as a sedative during the implantation of a neurostimulator was found to be significantly higher compared to those who received propofol. In addition, dexmedetomidine provided an easier arousability than propofol. Given these differences, we were interested in whether better sedation and easier arousability would lead to better long-term effects of the neurostimulation procedure in the dexmedetomidine group, possibly due to a more targeted placement of the leads in this group.

In this follow-up trial, performed in January and February 2020, no significant differences were found between the dexmedetomidine group and the propofol group regarding all outcome parameters (ie, global perceived effect, pain course, complaints of anxiety and depression, physical functioning, and treatment course). Nevertheless, it is notable that more surgical site pain occurred in the dexmedetomidine group than the propofol group (five vs zero patients with surgical site pain). We have no plausible explanation for this other than this result is coincidental.

Furthermore, an association between greater patient satisfaction with sedation (subscale procedural recall) and greater satisfaction reported at follow-up is reported. Since this association is a trend $(\mathrm{P}>0.05<0.10)$ a sturdy appreciation of this association is not justified in our opinion. This is due to the fact that no correction for multiple comparisons has been made, which inherently leads to an increased chance of erroneous findings.

In addition, it is worth mentioning that patients in both groups remain, even after a relatively long time, quite satisfied with the neurostimulation treatment (median satisfaction score 2.0 [IQR 2.0-3.0]), which means that they on average were very satisfied (see the classification in Table 1).

\section{Limitations}

Several limitations of this study should be mentioned. This was a retrospective cohort study in which the results could be biased by patients' memory and a possible response shift leading to a reduced internal validity. The more so, given the relatively long period between the implantation and the completion of the questionnaire. The statistical power was most likely negatively affected due to the many exclusions. However, we have no reason to assume that the missings were selective since they were equally spread across the groups. Furthermore, data from the medical charts were lost during the follow-up because they had not been recorded in a standardised manner and some data were missing. In addition, we did not adjust the error rate $\alpha$ for multiple testing because it would have reduced the power of the tests even more.

\section{Conclusions}

Regarding the long-term efficacy of neurostimulation treatment, based on the indirect influence of better sedation resulting in better lead placement during a paresthesia based implantation, in terms of global perceived effect, pain course, and physical and emotional functioning, no statistically significant differences were found between the dexmedetomidine and the propofol group. We found a trend towards greater satisfaction (subscale of GPE) in the dexmedetomidine group, compared to the propofol group. A correlation was found between patient satisfaction with sedation during 
neurostimulation intervention (side effects and procedural recall) and satisfaction with the neurostimulation treatment at follow-up.

\section{Ethical Approval}

Research involving human subjects complied with all relevant national regulations, institutional policies and is in accordance with the tenets of the Helsinki Declaration (as amended in 2013), and has been approved by the authors' Institutional Review Board or equivalent research ethical committee (MEC-2018-1267).

\section{Author Contributions}

All authors made a significant contribution to the work reported, whether that is in the conception, study design, execution, acquisition of data, analysis and interpretation, or in all these areas; took part in drafting, revising or critically reviewing the article; gave final approval of the version to be published; have agreed on the journal to which the article has been submitted; and agree to be accountable for all aspects of the work.

\section{Funding}

Drs. ter Bruggen was supported by institutional sources and a grant [grant 16-1444] from the "Stichting Erasmus Fonds Pijnbestrijding" located in Rotterdam, the Netherlands.

\section{Disclosure}

Professor Frank JPM Huygen reports personal fees from ABBOTT, Grunenthal, Pfizer, and Saluda, outside the submitted work. The authors report no other conflicts of interest in this work.

\section{References}

1. Simpson EL, Duenas A, Holmes MW, Papaioannou D, Chilcott J. Spinal cord stimulation for chronic pain of neuropathic or ischaemic origin: systematic review and economic evaluation. Health Technol Assess. 2009;13(17):iii, ix-x, 1-154. doi:10.3310/hta13170
2. Budd K. Spinal cord stimulation: cost-benefit study. Neuromodulation. 2002;5(2):75-78. doi:10.1046/j.1525-1403.2002.02012.x

3. Elsamadicy AA, Yang S, Sergesketter AR, et al. Prevalence and cost analysis of Complex Regional Pain Syndrome (CRPS): a role for neuromodulation. Neuromodulation. 2018;21(5):423-430. doi:10.1111/ner.12691

4. Falowski SM, Celii A, Sestokas AK, Schwartz DM, Matsumoto C, Sharan A. Awake vs. asleep placement of spinal cord stimulators: a cohort analysis of complications associated with placement. Neuromodulation. 2011;14(2):130-135. doi:10.1111/j.15251403.2010.00319.X

5. Kapural L, Yu C, Doust MW, et al. Comparison of $10-\mathrm{kHz}$ high-frequency and traditional low-frequency spinal cord stimulation for the treatment of chronic back and leg pain: 24-month results from a multicenter, randomized, controlled pivotal trial. Neurosurgery. 2016;79(5):667-677. doi:10.1227/NEU.0000000000001418

6. Ter Bruggen F, Stronks DL, Huygen F. Survey on sedation-analgesia regimens, in particular the use of dexmedetomidine, among Dutch implanters of spinal cord neurostimulators. Scand J Pain. 2019;19 (4):823-827. doi:10.1515/sjpain-2019-0058

7. Ter Bruggen F, Stronks DL, Huygen F. Cost analysis of dexmedetomidine versus propofol during the implantation of a neurostimulator. Acta Anaesthesiol Scand. 2020;64(6):861-862. doi:10.1111/aas.13579

8. Ter Bruggen FF, Eralp I, Leliveld L, Jansen C, Stronks DL, Huygen FJ. Dexmedetomidine as a sedative in the awake implantation of a neuromodulative system. Pain Pract. 2017;17(2):208-213. doi:10.1111/papr.12425

9. Vanhauwaert DJ, Couvreur T, Vandebroek A, De Coster O, Hanssens K. Conscious sedation using dexmedetomidine during surgical paddle lead placement improves outcome in spinal cord stimulation: a case series of 25 consecutive patients. Neuromodulation. 2020. doi:10.1111/ner.13124

10. Goettel N, Bharadwaj S, Venkatraghavan L, Mehta J, Bernstein M, Manninen PH. Dexmedetomidine vs propofol-remifentanil conscious sedation for awake craniotomy: a prospective randomized controlled trial. Br J Anaesth. 2016;116(6):811-821. doi:10.1093/bja/aew024

11. Ter Bruggen F, Ceuppens C, Leliveld L, Stronks DL, Huygen F. Dexmedetomidine vs propofol as sedation for implantation of neurostimulators: a single-center single-blinded randomized controlled trial. Acta Anaesthesiol Scand. 2019;63(10):1321-1329. doi:10.1111/aas.13452

12. Hudak PL, Wright JG. The characteristics of patient satisfaction measures. Spine. 2000;25(24):3167-3177. doi:10.1097/00007632200012150-00012

13. Pope JE, Deer TR, Falowski S, et al. Multicenter Retrospective Study of neurostimulation with exit of therapy by explant. Neuromodulation. 2017;20(6):543-552. doi:10.1111/ner.12634
Journal of Pain Research

\section{Publish your work in this journal}

The Journal of Pain Research is an international, peer reviewed, open access, online journal that welcomes laboratory and clinical findings in the fields of pain research and the prevention and management of pain. Original research, reviews, symposium reports, hypothesis formation and commentaries are all considered for publication. The manuscript management system is completely online and includes a very quick and fair peer-review system, which is all easy to use. Visit http:// www.dovepress.com/testimonials.php to read real quotes from published authors. 